\title{
Toe-brachial index as a predictor of cardiovascular disease and all-cause mortality in people with type 2 diabetes and microalbuminuria
}

\author{
Emilie H. Zobel ${ }^{1}$ • Bernt Johan von Scholten ${ }^{1} \cdot$ Henrik Reinhard ${ }^{1}$. \\ Frederik Persson $^{1} \cdot$ Tine W. Hansen ${ }^{1}$ - Hans-Henrik Parving ${ }^{2}$. \\ Peter K. Jacobsen ${ }^{3}$ Peter Rossing ${ }^{1,4,5}$
}

Received: 2 February 2017 / Accepted: 22 May 2017 /Published online: 5 July 2017

(C) Springer-Verlag GmbH Germany 2017

\begin{abstract}
Aims/hypothesis The study aimed to evaluate toe-brachial index (TBI) and ankle-brachial index (ABI) as determinants of incident cardiovascular disease (CVD) and all-cause mortality in people with type 2 diabetes and microalbuminuria.

Methods This was a prospective study including 200 participants. Unadjusted and adjusted (traditional risk factors and additional inclusion of $\mathrm{N}$-terminal pro-brain natriuretic peptide [NT-proBNP] and coronary artery calcification) Cox regression models were performed. C statistics and relative integrated discrimination improvement (rIDI) evaluated risk prediction improvement.

Results Median follow-up was 6.1 years; 40 CVD events and 26 deaths were recorded. Lower TBI was associated with increased risk of CVD (HR per 1 SD decrease: 1.55 [95\% CI $1.38,1.68])$ and all-cause mortality $(1.41[1.22,1.60])$ unadjusted and after adjustment for traditional risk factors (CVD $1.50[1.27,1.65]$ and all-cause mortality $1.37[1.01,1.60])$.
\end{abstract}

Electronic supplementary material The online version of this article (doi:10.1007/s00125-017-4344-x) contains peer-reviewed but unedited supplementary material, which is available to authorised users.

Emilie H. Zobel

emilie.hein.zobel@ regionh.dk

1 Steno Diabetes Center Copenhagen, Niels Steensens Vej 2, 2820 Gentofte, Denmark

2 Department of Endocrinology, Rigshospitalet, University of Copenhagen, Copenhagen, Denmark

3 The Heart Center, Rigshospitalet, University of Copenhagen, Copenhagen, Denmark

4 University of Copenhagen, Copenhagen, Denmark

5 Faculty of Health, Aarhus University, Aarhus, Denmark
Lower ABI was a determinant of CVD $(1.49[1.32,1.61])$ and all-cause mortality $(1.37[1.09,1.57])$ unadjusted and after adjustment for traditional risk factors (CVD 1.44 [1.23, 1.59] and all-cause mortality $1.39[1.07,1.60])$. After additional adjustment for NT-proBNP and coronary artery calcification, lower TBI remained a determinant of CVD $(p=0.023)$. When TBI was added to traditional risk factors, the AUC increased significantly for CVD, by 0.063 (95\% CI $0.012,0.115)$ from 0.743 ( $p=0.016$ ), but not for all-cause mortality; adding ABI did not improve the AUC significantly. The rIDI for TBI was $46.7 \%(p<0.001)$ for CVD and $46.0 \%(p=0.002)$ for allcause mortality; for ABI, the rIDI was $51.8 \%$ ( $p=0.004$ ) for CVD and $53.6 \%(p=0.031)$ for all-cause mortality.

Conclusions/interpretation Reduced TBI and ABI were associated with increased risk of CVD and all-cause mortality, independent of traditional risk factors in type 2 diabetes, and improved prognostic accuracy.

Keywords Ankle-brachial index · Cardiovascular disease . Carotid intima-media thickness $\cdot$ Microalbuminuria . Peripheral arterial disease $\cdot$ Toe-brachial index $\cdot$ Type 2 diabetes

\begin{tabular}{|c|c|}
\hline \multicolumn{2}{|c|}{ Abbreviations } \\
\hline $\mathrm{ABI}$ & Ankle-brachial index \\
\hline CVD & Cardiovascular disease \\
\hline IDI & $\begin{array}{l}\text { Integrated discrimination } \\
\text { improvement }\end{array}$ \\
\hline IQR & Interquartile range \\
\hline NT-proBNP & $\mathrm{N}$-terminal pro-brain natriuretic peptide \\
\hline rIDI & $\begin{array}{l}\text { Relative integrated discrimination } \\
\text { improvement }\end{array}$ \\
\hline ROC & Receiver operating characteristic \\
\hline
\end{tabular}


TBI Toe-brachial index

UAER Urinary AER

\section{Introduction}

Cardiovascular disease (CVD) is among the leading causes of morbidity and mortality in type 2 diabetes, and accurate risk stratification of these high-risk individuals is important. Currently, risk prediction is often based on established cardiovascular risk factors. However, there is increasing recognition of the imprecision in relying only on these risk factors, and cardiovascular risk prediction may be improved by adding a measure of atherosclerosis.

Assessment of toe-brachial index (TBI) and ankle-brachial index $(\mathrm{ABI})$ is non-invasive, inexpensive and easily performed in the clinical setting $[1,2]$. TBI and ABI are diagnostic tests for peripheral arterial disease and prognostic for healing of foot ulcers; they are also markers of systemic atherosclerosis and thus could improve the identification of highrisk individuals.

Current evidence of the value of TBI in risk stratification in type 2 diabetes is sparse. Two recent studies investigated the predictive role of TBI in people with type 2 diabetes; in 81 participants included after acute coronary syndrome, low TBI $(<0.6)$ was associated with increased risk of recurrent CVD [3]. A study with 17.2 years of follow-up, including a subgroup of 155 type 2 diabetic individuals, found no association between TBI and cardiovascular or all-cause mortality after adjustment [4].

ABI has been shown to predict cardiovascular morbidity and mortality in the general population [5] and in type 2 diabetes [6]. However, the validity of ABI in diabetes is still debated as people with diabetes often have medial arterial calcification, which might cause false elevated or false normal ankle pressure $[7,8]$. The vessels in the toe are less susceptible to calcification; thus, TBI may only be slightly affected by calcification and therefore perform as a better marker of atherosclerosis compared with ABI [9].

Measurement of carotid intima-media thickness is also a non-invasive examination of atherosclerosis, but needs to be performed by a trained technician in designated clinical settings. Conflicting results have been published on the added value of carotid intima-media thickness in cardiovascular risk prediction in the general population [10] and in type 2 diabetes [11].

The aim of this prospective study was to determine the added value of TBI and ABI as well as carotid intima-media thickness in risk prediction for CVD and all-cause mortality in individuals with type 2 diabetes and microalbuminuria, but without the clinical features of coronary artery disease.

As has been recently published, N-terminal pro-brain natriuretic peptide (NT-proBNP) level and coronary artery calcification score are powerful predictors of outcome, but are also complicated and expensive to assess [12]. We investigated the added value of TBI, ABI and carotid intima-media thickness to the traditional risk factors with and without NTproBNP level and coronary artery calcification score.

\section{Methods}

\section{Participants and study procedures}

In 2007-2008, we identified a cohort of 200 people with type 2 diabetes treated in a secondary care setting at the Steno Diabetes Center Copenhagen, Gentofte, Denmark. The selection process has previously been described in detail [13]. This follow-up was described in the original study protocol and members of the original study group are authors of this paper. Participants had type 2 diabetes according to WHO criteria without history or symptoms suggestive of coronary artery disease or other cardiac disease (assessed from medical records, interviews and questionnaires). Participants had persistent (two out of three consecutive measurements) urinary AER (UAER) $>30 \mathrm{mg} / 24 \mathrm{~h}$. Participants received multifactorial intervention (glycaemic, lipid and blood pressure control, anti-thrombotic therapy and lifestyle modification) in line with the findings from the Steno-2 Study [14].

The study complied with the Declaration of Helsinki. The research protocol was approved by the local ethics committee and all participants gave written informed consent.

\section{Investigations of the peripheral and coronary arteries}

Systolic blood pressure in the ankle and first toe were measured on both legs by the strain gauge technique and the lowest pressures were used for calculation of TBI and ABI, respectively [15]. Measurements were taken in a temperaturecontrolled room after participants had rested for $10 \mathrm{~min}$ in the supine position. The reliability of TBI and ABI has been evaluated in previous studies. The reproducibility of ABI is between 0.10 and 0.15 . For TBI, the variability is greater, ranging from the same to about twice that of ABI [16]. Brachial blood pressure was measured in the dominant arm with an appropriately sized cuff after $10 \mathrm{~min}$ rest in the supine position. Two measurements were obtained and averaged. Baseline measures of TBI and ABI were available for 191 and 178 participants, respectively.

Carotid intima-media thickness was measured by an experienced technician at the posterior wall $20 \mathrm{~mm}$ proximal to the bifurcation of the common carotid artery bilaterally, taken as the distance between the luminal intima interface and the adventitial media interface (Acuson Cypress ultrasound scanner with a linear probe 7-10 MHz 7L3, Siemens, OH, USA). Carotid intima-media thickness was measured on both sides 
and averaged [17]. Thickness measurements were available for 182 participants.

Coronary artery calcification scanning was performed as previously described [13]. For each participant, the total Agatston coronary artery calcification score was determined, including intimal and medial calcification of the left main, left anterior descending artery, circumflex artery and right coronary artery.

\section{Biochemical and other analyses}

UAER was measured in $24 \mathrm{~h}$ urine samples using an enzyme immunoassay (Vitros, Raritan, NJ, USA). Plasma NT-proBNP was analysed by immunoassay, as previously described [18]. Current smoking was defined as one or more cigarettes, cigars or pipes per day.

\section{Follow-up}

All participants were traced on 1 January 2014 through the Danish National Death Register and the Danish National Health Register to obtain information on cause of death and hospital admissions as previously described in detail [12]. Unless an unequivocal non-cardiovascular cause was established, all deaths were classified as cardiovascular. No participants were lost to follow-up.

The primary endpoint consisted of cardiovascular mortality, non-fatal myocardial infarction (ICD-10 codes I20-I25); (www.who.int/classifications/icd/en/), stroke (ICD-10 codes I61 or I63), ischaemic CVD (ICD-10 code I70) and heart failure (ICD-10 code I50). The analysis included only the first endpoint for participants who experienced multiple endpoints. The secondary endpoint was all-cause mortality.

\section{Statistical analyses}

Non-normally distributed variables (UAER, NT-proBNP, coronary artery calcification score) are reported as median with interquartile range (IQR), other continuous variables as means $\pm \mathrm{SD}$; categorical variables are summarised as total numbers with corresponding percentages.

The correlations between measures of peripheral arterial disease, coronary artery calcification score and carotid intima-media thickness were calculated from unadjusted and adjusted linear regression models and presented as standardised $\beta$ coefficients. Adjustment included sex, age, systolic blood pressure, LDL-cholesterol, smoking, $\mathrm{HbA}_{1 \mathrm{c}}$, plasma creatinine and UAER.

We applied Cox regression models to estimate the HRs with $95 \%$ CIs for the CVD endpoint and all-cause mortality per $1 \mathrm{SD}$ decrease in TBI, ABI and carotid intima-media thickness. TBI and ABI were also analysed as categorical variables using established cut-off points [19-21]. In contrast to the well-defined and evidence-based limits of the ABI, the diagnostic criterion for a pathologic TBI remains ambiguous and is not strictly evidence based [1].

Carotid intima-media thickness was not dichotomised as no cut-off point is established.

First, we investigated whether any association existed between TBI, ABI and carotid intima-media thickness and our two pre-defined endpoints in the unadjusted model (Model 1). Subsequent adjustment included traditional cardiovascular risk factors based on existing evidence: sex, age, systolic blood pressure, LDL-cholesterol, smoking, $\mathrm{HbA}_{1 \mathrm{c}}$, plasma creatinine and UAER (Model 2). A third model included these traditional cardiovascular risk factors plus NT-proBNP level and coronary artery calcification score (Model 3). KaplanMeier failure function was applied to compare the risks of the CVD endpoint and all-cause mortality according to TBI (above or below 0.64) and ABI (above or below 0.9) at baseline.

Next, we calculated the potential additional predictive ability of TBI and ABI over traditional cardiovascular risk factors using receiver operating characteristic (ROC) curves based on logistic regression models. The AUC was calculated in a model including only the traditional cardiovascular risk factors, and in the same model with TBI or ABI added.

We calculated the integrated discrimination improvement (IDI) statistics between the model including traditional cardiovascular risk factors vs the new model including traditional cardiovascular risk factors plus TBI or ABI. IDI assesses the ability of a new marker to improve the average sensitivity without sacrificing average specificity. Compared with AUC, the IDI may be a more powerful method to demonstrate improved diagnostic performance [22]. Relative IDI (rIDI) is provided, reported as a percentage, for ease of interpretation of the contribution of TBI and ABI. rIDI is defined as the increase in discrimination slope divided by the slope of the model including only the traditional cardiovascular risk factors [22].

Only participants with TBI $(n=193)$ and ABI $(n=179)$ baseline measurements available were included in the statistical analyses. A two-tailed $p$ value $<0.05$ was considered significant. Statistical analyses were performed using SPSS for Windows (version 23.0, Chicago, IL, USA), SAS software (version 9.3, SAS Institute, Cary, NC, USA) and Stata/IC version 14.1 for Windows (StataCorp, College Station, TX, USA).

\section{Results}

\section{Participant characteristics}

A total of 200 participants were included in the study. Of these, $151(76 \%)$ were men, mean \pm SD age was $59 \pm 9$ years 
and mean diabetes duration was $13 \pm 7$ years. Most participants were treated with oral glucose-lowering agents, insulin and cardiovascular medication (anti-hypertensive drugs [99\%], renin-angiotensin-aldosterone system-blocking treatment [98\%], statins [95\%] and aspirin [90\%]). Peripheral arterial disease, defined as $\mathrm{ABI}<90$ and/or TBI $<0.64$ and/or prior surgery for peripheral arterial disease, was demonstrated in $20 \%$ of the participants at baseline. A higher detection rate for peripheral arterial disease was found applying TBI $<0.64$ compared with $\mathrm{ABI}<0.9$ (15\% vs $11 \%)$.

Table 1 lists the baseline characteristics of participants, categorised according to TBI below or above 0.64 and ABI below or above 0.90 .

The unadjusted and adjusted correlations between measures of peripheral arterial disease, coronary artery calcification score and carotid intima-media thickness are shown in Table 2 .

\section{Incidence of the CVD endpoint and mortality}

During the follow-up period (median [IQR] 6.1 [5.96.6 years]) 40 participants experienced at least one event from the combined cardiovascular endpoint: 11 cardiovascular events were fatal (two cases of acute myocardial infarction, one case of ischaemic CVD, six sudden and otherwise unexplained deaths and two cases of heart failure) and 29 cardiovascular events were non-fatal events leading to hospital admission (three cases of acute myocardial infarction, three strokes, 19 cases of ischaemic CVD and four cases of heart failure); 26 participants died (11 deaths were classified as CVD related, nine as cancer related and six as related to other causes).

\section{TBI as a risk marker}

Table 3 shows the associations for TBI with the composite cardiovascular endpoint and all-cause mortality with TBI modelled as a continuous and a binary variable.

Determinant modelled as a continuous variable In the crude analysis (Model 1), lower TBI was associated with higher risk of both the composite cardiovascular endpoint and all-cause mortality (Table 3). In Model 2, TBI remained associated with both endpoints (Table 3).

In Model 3 (Model 2 plus NT-proBNP and coronary artery calcification), TBI remained associated with the composite cardiovascular endpoint, but not with all-cause mortality (Table 3).

As shown in Table 4, in relation to the composite cardiovascular endpoint, the AUC increased from 0.743 (95\% CI $0.661,0.825)$ to $0.806(0.730,0.882)$ after adding TBI to the model including traditional risk factors. The improvement in AUC was significant $(0.063[0.012,0.115])$. In relation to allcause mortality, the AUC increased from $0.736(0.632,0.840)$ to $0.752(0.639,0.865)$ after adding TBI to the model including traditional risk factors. The improvement in AUC was $0.016(-0.055,0.088)$.

The rIDI for TBI was $46.7 \%(p<0.001)$ for the cardiovascular endpoint, and $46.0 \%(p=0.002)$ in relation to all-cause mortality.

Determinant modelled as a binary variable The cumulative incidence of the composite cardiovascular endpoint and allcause mortality was higher in participants with $\mathrm{TBI}<0.64$ (Table 3 and Fig. 1). In Model 2, TBI $<0.64$ remained associated with the composite cardiovascular endpoint and allcause mortality (Table 3). After further adjustment (Model $3)$, TBI was no longer significant for the cardiovascular endpoint, but the risk of all-cause mortality remained higher in participants with TBI $<0.64$ (Table 3).

\section{$\mathrm{ABI}$ as a risk marker}

Table 3 shows the associations for ABI with the composite cardiovascular endpoint and all-cause mortality with $\mathrm{ABI}$ modelled as a continuous and a binary variable.

Determinant modelled as a continuous variable In the crude analysis (Model 1), lower ABI was associated with higher risk of both the composite cardiovascular endpoint and all-cause mortality (Table 3). In Model 2, ABI remained associated with both endpoints. However, in Model 3 (Model 2 plus NT-proBNP and coronary artery calcification), ABI was no longer significant for the two endpoints (Table 3).

As shown in Table 4, in relation to the composite cardiovascular endpoint, the AUC increased from $0.761(95 \% \mathrm{CI}$ $0.670,0.852)$ to $0.816(0.725,0.908)$ after adding $\mathrm{ABI}$ to the model including traditional risk factors. The improvement in AUC was $0.055(-0.003,0.113)$. In relation to all-cause mortality, the AUC increased from $0.746(0.628,0.865)$ to 0.766 $(0.639,0.894)$ after adding $\mathrm{ABI}$ to the model including traditional risk factors. The improvement in AUC was 0.020 $(-0.059,0.099)$.

The rIDI for ABI was $51.8 \%(p=0.004)$ in relation to the cardiovascular endpoint and $53.6 \%(p=0.031)$ for all-cause mortality.

Determinant modelled as a binary variable The cumulative incidence of the composite cardiovascular endpoint and allcause mortality was higher in participants with $\mathrm{ABI}<0.90$ (Table 3 and Fig. 1). In Model 2, ABI $<0.90$ remained associated with both endpoints (Table 3). However, in Model 3, after comprehensive adjustment, $\mathrm{ABI}<0.90$ was not associated with the risk of CVD or all-cause mortality (Table 3). 
Table 1 Clinical characteristics of the study population at baseline categorised according to TBI below or above 0.64 and ABI below or above 0.90

\begin{tabular}{|c|c|c|c|c|}
\hline \multirow[t]{2}{*}{ Characteristic } & \multicolumn{2}{|l|}{ TBI } & \multicolumn{2}{|l|}{$\mathrm{ABI}$} \\
\hline & $(<0.64 ; n=30)$ & $(\geq 0.64 ; n=163)$ & $(<0.90 ; n=22)$ & $(\geq 0.90 ; n=157)$ \\
\hline Male, $n(\%)$ & $25(83)$ & $119(74)$ & $19(86)$ & $115(74)$ \\
\hline Age (years) & $63 \pm 6$ & $58 \pm 9$ & $63 \pm 4$ & $58 \pm 9$ \\
\hline Known duration of diabetes (years) & $16 \pm 7$ & $12 \pm 7$ & $15 \pm 7$ & $12 \pm 7$ \\
\hline BMI $\left(\mathrm{kg} / \mathrm{m}^{2}\right)$ & $31 \pm 5$ & $33 \pm 6$ & $30 \pm 5$ & $32 \pm 6$ \\
\hline $\mathrm{HbA}_{1 \mathrm{c}}(\%)$ & $7.9 \pm 1.3$ & $7.9 \pm 1.4$ & $8.0 \pm 1.1$ & $7.8 \pm 1.4$ \\
\hline $\mathrm{HbA}_{1 \mathrm{c}}(\mathrm{mmol} / \mathrm{mol})$ & $63 \pm 14.2$ & $63 \pm 15.3$ & $64 \pm 12.0$ & $62 \pm 15.3$ \\
\hline UAER (mg/24 h) & $123(46-214)$ & $93(38-230)$ & $153(75-541)$ & $95(38-223)$ \\
\hline P-creatinine $(\mu \mathrm{mol} / \mathrm{l})$ & $84 \pm 16$ & $75 \pm 19$ & $76 \pm 16$ & $75 \pm 18$ \\
\hline eGFR ( $\left.\mathrm{ml} \mathrm{min} \min ^{-1} 1.73 \mathrm{~m}^{-2}\right)$ & $81 \pm 14$ & $91 \pm 18$ & $88 \pm 15$ & $91 \pm 18$ \\
\hline Systolic blood pressure (mmHg) & $131 \pm 16$ & $129 \pm 16$ & $131 \pm 19$ & $129 \pm 16$ \\
\hline LDL-cholesterol (mmol/l) & $2.0 \pm 0.7$ & $1.8 \pm 0.8$ & $2.2 \pm 0.9$ & $1.8 \pm 0.8$ \\
\hline Current smoker, $n(\%)$ & $9(30)$ & $47(29)$ & $7(32)$ & $45(29)$ \\
\hline NT-proBNP (ng/l) & $59.4(26.3-165.2)$ & $45.8(16.9-88.8)$ & $59.4(28.3-179.6)$ & $42.4(15.3-88.5)$ \\
\hline Coronary artery calcium score (Agatston units) & $545(199-1207)$ & $130(2-478)$ & $679(224-1971)$ & $94(1-433)$ \\
\hline Carotid intima-media thickness $(\mathrm{mm})$ & $0.8 \pm 0.1$ & $0.7 \pm 0.1$ & $0.8 \pm 0.1$ & $0.7 \pm 0.2$ \\
\hline \multicolumn{5}{|l|}{ Treatment with: } \\
\hline Oral glucose-lowering agents, $n(\%)$ & $23(77)$ & $140(87)$ & $18(82)$ & $132(85)$ \\
\hline Insulin, $n(\%)$ & $21(70)$ & $98(61)$ & $16(73)$ & $91(58)$ \\
\hline Anti-hypertensive drugs, $n(\%)$ & $30(100)$ & $161(100)$ & $22(100)$ & $155(99)$ \\
\hline Statin, $n(\%)$ & $28(93)$ & $155(96)$ & $19(86)$ & $149(96)$ \\
\hline Aspirin, $n(\%)$ & $27(90)$ & $148(92)$ & $21(95)$ & $141(90)$ \\
\hline
\end{tabular}

Non-normally distributed variables (UAER, NT-proBNP, coronary artery calcification score) are reported as median with IQR; other continuous variables as means $\pm \mathrm{SD}$

Categorical variables are shown as total numbers with corresponding percentages

\section{Sensitivity analyses}

The conclusions were unaffected after exclusion of people with $\mathrm{ABI}>1.3$ when applying 0.70 as cut-off for TBI and

Table 2 Correlations between measures of peripheral arterial disease, coronary artery calcification score and carotid intima-media thickness

\begin{tabular}{lll}
\hline Measurement & TBI & ABI \\
\hline Coronary artery calcification & \\
$\quad$ Unadjusted & $-0.39(-0.25,-0.52)^{* * *}$ & $-0.36(-0.21,-0.50)^{* * *}$ \\
Adjusted & $-0.27(-0.10,-0.43)^{* *}$ & $-0.33(-0.15,-0.49)^{* * *}$ \\
Carotid intima-media thickness & \\
Unadjusted & $-0.22(-0.07,-0.37)^{* *}$ & $-0.26(-0.11,-0.42)^{* *}$ \\
Adjusted & $-0.12(-0.27,0.03)$ & $-0.21(-0.05,-0.37)^{*}$ \\
\hline
\end{tabular}

Values represent standardised $\beta$ coefficients with $95 \%$ CIs

Adjustment included sex, age, systolic blood pressure, LDL-cholesterol, smoking, $\mathrm{HbA}_{1 \mathrm{c}}$, plasma creatinine and urinary albumin excretion rate ${ }^{*} p<0.05,{ }^{* *} p<0.01$ and ${ }^{* * *} p<0.001$ for correlations between TBI and coronary artery calcification and carotid intima-media thickness, respectively; and correlation between $\mathrm{ABI}$ and coronary artery calcification and carotid intima-media thickness, respectively. in analyses omitting adjustment for systolic blood pressure (electronic supplementary material [ESM] Table 1).

\section{Carotid intima-media thickness as a risk marker}

Table 3 shows the associations between carotid intima-media thickness and the composite cardiovascular endpoint and allcause mortality with determinant modelled as a continuous variable.

In the unadjusted analysis (Model 1), lower carotid intimamedia thickness was associated with lower risk of the composite cardiovascular endpoint and all-cause mortality (Table 3). In Model 2, carotid intima-media thickness was not associated with the composite cardiovascular endpoint or all-cause mortality (Table 3).

\section{Discussion}

The current prospective study, including 200 individuals with type 2 diabetes, microalbuminuria and no clinical features of coronary artery disease followed for 6 years, demonstrates TBI 
Table 3 TBI and ABI in relation to risk of fatal and non-fatal cardiovascular events and all-cause mortality

\begin{tabular}{|c|c|c|c|c|}
\hline \multirow[t]{2}{*}{ Measurement/model } & \multicolumn{2}{|c|}{ Cardiovascular events $(n=40)$} & \multicolumn{2}{|c|}{ All-cause mortality $(n=26)$} \\
\hline & HR $(95 \%$ CI) & $p$ value & $\mathrm{HR}(95 \% \mathrm{CI})$ & $p$ value \\
\hline \multicolumn{5}{|c|}{$\mathrm{TBI}(n=193 ; \mathrm{SD}=0.23)$} \\
\hline 1 & $1.55(1.38,1.68)$ & $<0.0001$ & $1.41(1.22,1.60)$ & 0.010 \\
\hline 2 & $1.50(1.27,1.65)$ & $<0.0001$ & $1.37(1.01,1.60)$ & 0.049 \\
\hline 3 & $1.36(1.06,1.56)$ & 0.023 & $1.24(0.78,1.53)$ & 0.25 \\
\hline \multicolumn{5}{|c|}{$\mathrm{ABI}(n=179 ; \mathrm{SD}=0.17)$} \\
\hline 1 & $1.49(1.32,1.61)$ & $<0.0001$ & $1.37(1.09,1.57)$ & 0.015 \\
\hline 2 & $1.44(1.23,1.59)$ & $<0.0001$ & $1.39(1.07,1.60)$ & 0.021 \\
\hline 3 & $1.27(0.95,1.48)$ & 0.087 & $1.27(0.81,1.55)$ & 0.20 \\
\hline \multicolumn{5}{|c|}{ Carotid intima-media thickness $(n=182 ; \mathrm{SD}=0.15 \mathrm{~mm})$} \\
\hline 1 & $0.63(0.19,0.96)$ & 0.028 & $0.48(0.01,0.88)$ & 0.008 \\
\hline 2 & $0.93(0.53,1.22)$ & 0.68 & $0.64(0.06,1.05)$ & 0.096 \\
\hline 3 & $0.85(0.46,1.19)$ & 0.43 & $0.60(0.01,1.06)$ & 0.097 \\
\hline \multicolumn{5}{|l|}{ TBI $(<0.64 ; n=30)$} \\
\hline 1 & $3.4(1.7,6.9)$ & $<0.0001$ & $3.8(1.6,8.8)$ & 0.002 \\
\hline 2 & $2.5(1.2,5.2)$ & 0.016 & $3.5(1.4,8.6)$ & 0.008 \\
\hline 3 & $1.8(0.8,4.0)$ & 0.18 & $3.2(1.2,8.5)$ & 0.017 \\
\hline \multicolumn{5}{|l|}{$\mathrm{ABI}(<0.90 ; n=22)$} \\
\hline 1 & $4.9(2.2,10.5)$ & $<0.0001$ & $3.9(1.5,10.3)$ & 0.007 \\
\hline 2 & $3.2(1.4,7.5)$ & 0.007 & $3.7(1.3,10.6)$ & 0.002 \\
\hline 3 & $2.1(0.9,5.1)$ & 0.10 & $3.2(1.0,10.8)$ & 0.058 \\
\hline
\end{tabular}

HRs for the determinant modelled as a continuous variable represents risk related to $1 \mathrm{SD}$ decrease

HRs for the determinant modelled as a binary variable represents risk compared with TBI $\geq 0.64(n=163)$ or ABI $\geq 0.90(n=157)$ group Model 1 is unadjusted; Model 2 is adjusted for sex, age, systolic blood pressure, LDL-cholesterol, smoking, HbA 1 , plasma creatinine and UAER Model 3 is as Model 2, but with coronary artery calcium score and NT-proBNP added

and $\mathrm{ABI}$ as determinants of CVD and all-cause mortality after adjustment for traditional cardiovascular risk factors. After additional adjustment for NT-proBNP level and coronary artery calcification score, lower TBI remained associated with CVD.
Analyses of the added discriminatory ability of TBI and $\mathrm{ABI}$ demonstrated a significant increase in the AUC for the cardiovascular endpoint when adding TBI to a model including traditional cardiovascular risk factors. The IDI statistics

Table 4 C statistics for TBI and ABI in relation to risk of fatal and non-fatal cardiovascular events and all-cause mortality

\begin{tabular}{lll}
\hline Panel/model & AUC $(95 \% \mathrm{CI})$ & Improvement AUC (95\% CI) \\
\hline $\begin{array}{l}\text { Panel a: composite CVD endpoint } \\
\text { Base model }\end{array}$ & $0.743(0.661,0.825)$ & $0.0632(0.0116,0.1147)$ \\
$\quad$ Base model + TBI & $0.806(0.730,0.882)$ & \\
Panel b: all-cause mortality & $0.736(0.632,0.840)$ & $0.0162(-0.0553,0.0876)$ \\
$\quad$ Base model & $0.752(0.639,0.865)$ & \\
Base model + TBI & $0.761(0.670,0.852)$ & $0.0553(-0.00270,0.113)$ \\
Panel c: composite CVD endpoint & $0.816(0.725,0.908)$ & \\
$\quad$ Base model & $0.746(0.628,0.865)$ & 0.066 \\
Base model + ABI & $0.766(0.639,0.894)$ & $0.0201(-0.0592,0.0993)$ \\
Panel d: all-cause mortality & & 0.62 \\
Base model & & \\
Base model + ABI & & \\
\hline
\end{tabular}

Base model includes sex, age, systolic blood pressure, LDL-cholesterol, smoking, $\mathrm{Hb}_{1 \mathrm{c}}$, plasma creatinine and UAER 
a

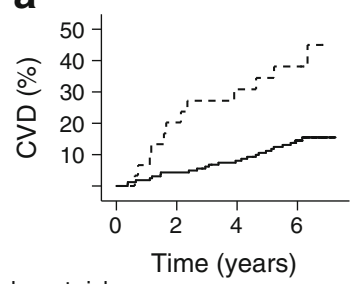

Number at risk

TBI $<64163155147 \quad 105$

$\mathrm{TBI} \geq 64 \begin{array}{llll}30 & 23 & 19 & 13\end{array}$

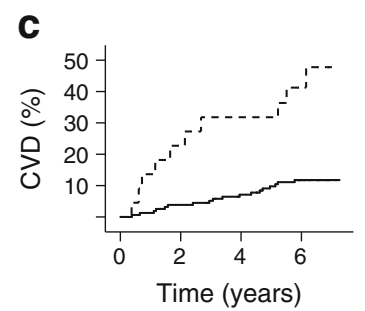

Number at risk

$\mathrm{ABI}<90157149141 \quad 102$

$A B I \geq 90 \quad 22 \quad 17 \quad 15 \quad 9$

\section{b}

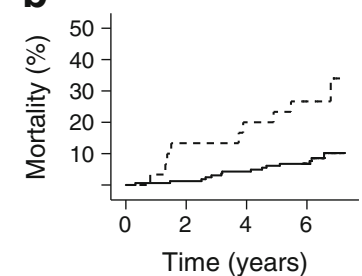

Number at risk

TBI $<64163161 \quad 156119$

TBI $\geq 6430 \quad 26 \quad 24 \quad 18$

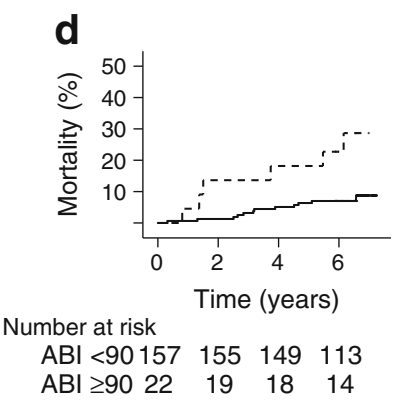

Fig. 1 Kaplan-Meier failure function estimates with two categories of TBI, above or below a cut-off of 0.64 , for (a) the composite cardiovascular endpoint (HR 3.4 [95\% CI 1.7, 6.9], $p<0.001$ ) and (b) all-cause mortality (3.8 [1.6, 8.8], $p=0.002)$, and with two categories of ABI, above or below cut-off of 0.9 , for (c) the composite cardiovascular endpoint (4.9 [2.2, 10.5], $p<0.001)$ and (d) all-cause mortality $(3.9$ [1.5, 10.3], $p=0.007)$. Numbers refer to participants in each category at risk at the beginning of each 2 year interval. Dashed line, below cut-off; solid line, above cut-off

show that adding TBI or ABI to traditional cardiovascular risk factors significantly improves the predictive capability for both CVD and all-cause mortality.

Recently, a study in 81 individuals with type 2 diabetes included after acute myocardial infarction and followed for 3.7 years demonstrated that low TBI $(<0.6)$ and low toe blood pressure $(<50 \mathrm{mmHg})$ predicted major cardiovascular events defined as a new episode of myocardial infarction, ischaemic cerebrovascular disease or peripheral arterial disease in appropriately adjusted models (low TBI, HR [95\% CI] 2.92 [1.04, 8.19]; low toe blood pressure 3.83 [1.45, 10.1]) [3]. A study including 155 type 2 diabetic and 469 non-diabetic participants demonstrated low ABI $(<0.90)$ to be associated with cardiovascular (HR [95\% CI] 2.57 [1.50, 4.40]) and allcause $(2.02[1.47,2.76])$ mortality after adjustment for risk factors included in the Framingham risk score. However, these associations were no longer significant (HR [95\% CI] 1.74 $[0.97,3.14]$ and $1.30[0.46,3.62])$ in analyses restricted to the diabetic subgroup [4]. The authors reported lower TBI to be associated with cardiovascular and all-cause mortality after adjustment for age, sex and glucose metabolism status, but the association was no longer significant after adjustment for the Framingham risk factors. Results for the predictive value of TBI restricted to the diabetic subgroup were not presented. In contrast to our study, individuals with prior CVD were

included and follow-up was longer (median 17.2 years). A study by Hyun et al demonstrated TBI $<0.40$ (TBI categories $<0.40,0.40-0.61,0.62-1.08$ and $>1.08$, with $0.62-1.08$ as the reference category) to predict high risk of cardiovascular mortality in analysis adjusted for traditional cardiovascular risk factors (HR [95\% CI] 2.25 [1.47, 3.43]). The study included 469 participants with clinically suspected atherosclerotic peripheral arterial disease, of whom 168 had diabetes; similar associations were demonstrated in the diabetic subgroup (2.28 [1.15, 4.55]). Median follow-up was 7.0 years [23].

Our present study provides support for the use of TBI as a tool refining risk prediction in type 2 diabetes. The addition of TBI to traditional cardiovascular risk factors improved risk prediction for CVD applying $\mathrm{C}$ statistics, and for CVD and all-cause mortality calculated as rIDI.

Recently, we demonstrated that a simple screening algorithm combining the NT-proBNP level and coronary artery calcification score added substantially to the risk information provided by traditional cardiovascular risk factors in the current cohort [12]. TBI remained associated with the composite cardiovascular endpoint even after adjustment for these powerful risk markers.

Our study expands on the existing literature by demonstrating that $\mathrm{ABI}$ - when modelled as a continuous and as a binary variable - was associated with incident CVD and all-cause mortality in asymptomatic people with type 2 diabetes and microalbuminuria independently of traditional risk factors. Continuous analysis has the greatest statistical power. However, cut-offs have greater applicability in the clinic.

The addition of ABI to traditional cardiovascular risk factors improved risk prediction for CVD and all-cause mortality evaluated by rIDI. The Fremantle Diabetes Study followed 1294 individuals with type 2 diabetes and previous CVD and demonstrated that low $(\leq 0.90)$ compared with normal (0.91-1.40) ABI was associated with cardiovascular mortality [6]. In 2368 people with type 2 diabetes and coronary artery disease included in the Bypass Angioplasty Revascularization Investigation 2 Diabetes Trial, low ABI $(<1.0)$ conferred an increased risk of mortality and major cardiovascular events during follow-up of a median 5.2 years [24].

Despite the existing literature, ABI has not been broadly implemented in daily practice for risk assessment in type 2 diabetes. This may be because of the reliability of ABI in people with diabetes in whom medial arterial calcification is frequently observed $[7,9,25]$. In a study including 83 people with diabetes (type $2,83 \%$ ) defined to be at high risk of peripheral arterial disease, examination with colour-flow duplex ultrasound demonstrated the presence of peripheral arterial occlusive disease in $57 \%$ of those with ABI between 0.9 and 1.3. This study confirmed that $\mathrm{ABI}$ often is incorrectly normal because of vessel stiffness in diabetes [7]. The vessels in the toe are less susceptible to medial calcification [9], therefore TBI may be more reliable for risk assessment in diabetes. 
However, the association was similar in a sub-analysis excluding participants with $\mathrm{ABI}>1.3$.

Measurement of carotid intima-media thickness has been proposed to improve risk assessment beyond established cardiovascular risk factors. However, a meta-analysis including 14 population-based cohorts ( 45,828 individuals) demonstrated that adding carotid intima-media thickness to the Framingham risk score was associated with only a small improvement in 10 year risk prediction of first-time myocardial infarction or stroke (increase in AUC from 0.757 to 0.759 ) [10]. Similarly, a retrospective study including 783 people with type 2 diabetes, but without a history of CVD, demonstrated that adding carotid intima-media thickness to Framingham risk score resulted in a small increase in AUC from 0.645 to 0.656 [11]. In our analysis, carotid intima-media thickness was associated with the development of the combined cardiovascular endpoint and all-cause mortality in the crude analysis, but these associations were no longer significant after adjustment for traditional cardiovascular risk factors. However, our study was much smaller and thus had less power. Our results suggest no added value of carotid intimamedia thickness measurements in cardiovascular risk prediction in type 2 diabetes. However, carotid intima-media thickness remains of interest in pathophysiological studies and as an endpoint in clinical trials.

The finding that both TBI and ABI were correlated with carotid intima-media thickness as well as coronary artery calcification illustrates that atherosclerosis is often a universal disease affecting different arterial beds. Despite this correlation, TBI was able to predict CVD in addition to the risk information provided by coronary artery calcification, further highlighting the strength of TBI.

\section{Clinical implications}

TBI is a relatively simple test easily performed in the clinical setting. Our findings of the added predictive value on top of traditional risk factors for both CVD and all-cause mortality highlight the great potential impact of implementing TBI measurement in type 2 diabetes in the clinical setting. Identifying individuals with the highest risk can enhance guidance of intensive management of vascular risk factors, including tighter goal setting for blood pressure, LDL-cholesterol and other cardiovascular risk factors.

\section{Strengths and limitations}

The strengths of our study include the prospective design, which included careful assessment of cardiovascular risk factors. This allowed extensive adjustment for these factors, including levels of NT-proBNP and coronary artery calcification score. Moreover, no participants were lost to follow-up. The significant results of the continuous models of ABI and TBI, without applying arbitrary thresholds, are other strong points.

Limitations include the relatively small sample size and that the study only included people with type 2 diabetes and microalbuminuria recruited from a single centre, limiting the generalisation of our findings. Individuals with microalbuminuria can be considered to be at a higher CVD risk and the lack of people with normoalbuminuria may have influenced the results.

Moreover, the inter- and intra-rater reliability of the ABI and TBI were not evaluated in our study. The larger variability of TBI might have impacted on the performance of TBI on the AUC models.

\section{Conclusion}

In people with type 2 diabetes and microalbuminuria but without clinical features of coronary artery disease, lower levels of TBI were associated with increased risk of CVD and all-cause mortality independently of traditional risk factors. After further adjustment for NT-proBNP level and coronary artery calcification score, lower TBI remained a significant predictor of CVD. The addition of TBI to traditional cardiovascular risk factors improved risk prediction for CVD and all-cause mortality. Lower ABI levels were associated with increased risk of CVD and all-cause mortality after adjustment for traditional cardiovascular risk factors, and improved risk prediction.

Acknowledgements We thank all the participants and acknowledge the work of L. Jelstrup (study nurse) and A. G. Lundgaard, B. R. Jensen, T. R. Juhl and J. A. Hermann (laboratory technicians), Steno Diabetes Center Copenhagen, Gentofte, Denmark. We thank J. Oellgaard, Department of Cardiology and Endocrinology, Slagelse Hospital, Slagelse, Denmark for preparing the figure for publication.

Data availability The datasets generated during and/or analysed during the current study are not publicly available because of data protection issues. An anonymised form is available from the corresponding author on reasonable request.

Funding The study was funded with a clinical research grant from the European Foundation for the Study of Diabetes (EFSD). Internal funding was provided by Steno Diabetes Center Copenhagen, Gentofte, Denmark.

Duality of interest statement The authors declare that there is no duality of interest associated with this manuscript.

Contribution statement EHZ, BJvS, HR, H-HP, PKJ and PR conceived and designed the research. EHZ, BJvS, FP, HR, PR and TWH analysed and interpreted the data. BJvS and TWH performed the statistical analysis and helped draft the manuscript. EHZ wrote the manuscript. FP, HR, H-HP, PKJ and PR critically revised the manuscript for key intellectual content. PR obtained funding and supervised the study. All 
authors approved the final version of the manuscript. EHZ is responsible for the integrity of the work as a whole.

\section{References}

1. Hoyer C, Sandermann J, Petersen LJ (2013) The toe-brachial index in the diagnosis of peripheral arterial disease. J Vasc Surg 58:231238

2. Jude EB, Eleftheriadou I, Tentolouris N (2010) Peripheral arterial disease in diabetes - a review. Diabet Med 27:4-14

3. Martin-Fuentes M, Herranz L, Saez-de-Ibarra L, Pallardo LF (2015) Low toe-brachial pressure index predicts increased risk of recurrent cardiovascular disease in type 2 diabetes. Diabetes Care 38:e53-e54

4. Hanssen NM, Huijberts MS, Schalkwijk CG, Nijpels G, Dekker JM, Stehouwer CD (2012) Associations between the anklebrachial index and cardiovascular and all-cause mortality are similar in individuals without and with type 2 diabetes: nineteen-year follow-up of a population-based cohort study. Diabetes Care 35: $1731-1735$

5. Ankle Brachial Index C, Fowkes FG, Murray GD et al (2008) Ankle brachial index combined with Framingham Risk Score to predict cardiovascular events and mortality: a meta-analysis. JAMA 300:197-208

6. Norman PE, Davis WA, Bruce DG, Davis TM (2006) Peripheral arterial disease and risk of cardiac death in type 2 diabetes: the Fremantle Diabetes Study. Diabetes Care 29:575-580

7. Potier L, Halbron M, Bouilloud F et al (2009) Ankle-to-brachial ratio index underestimates the prevalence of peripheral occlusive disease in diabetic patients at high risk for arterial disease. Diabetes Care 32:e44

8. Emanuele MA, Buchanan BJ, Abraira C (1981) Elevated leg systolic pressures and arterial calcification in diabetic occlusive vascular disease. Diabetes Care 4:289-292

9. Young MJ, Adams JE, Anderson GF, Boulton AJ, Cavanagh PR (1993) Medial arterial calcification in the feet of diabetic patients and matched non-diabetic control subjects. Diabetologia 36:615621

10. Den Ruijter HM, Peters SA, Anderson TJ et al (2012) Common carotid intima-media thickness measurements in cardiovascular risk prediction: a meta-analysis. JAMA 308:796-803

11. Yoshida M, Mita T, Yamamoto R et al (2012) Combination of the Framingham risk score and carotid intima-media thickness improves the prediction of cardiovascular events in patients with type 2 diabetes. Diabetes Care 35:178-180

12. von Scholten BJ, Reinhard H, Hansen TW et al (2015) Additive prognostic value of plasma $\mathrm{N}$-terminal pro-brain natriuretic peptide and coronary artery calcification for cardiovascular events and mortality in asymptomatic patients with type 2 diabetes. Cardiovasc Diabetol 14:59

13. Reinhard H, Hansen PR, Persson F et al (2011) Elevated NTproBNP and coronary calcium score in relation to coronary artery disease in asymptomatic type 2 diabetic patients with elevated urinary albumin excretion rate. Nephrol Dial Transplant 26:32423249

14. Gaede P, Lund-Andersen H, Parving HH, Pedersen O (2008) Effect of a multifactorial intervention on mortality in type 2 diabetes. N Engl J Med 358:580-591

15. Lassen NA, Tvedegaard E, Jeppesen FI, Nielsen PE, Bell G, Gundersen J (1972) Distal blood pressure measurement in occlusive arterial disease, strain gauge compared to xenon-133. Angiology 23:211-217

16. Bird CE, Criqui MH, Fronek A, Denenberg JO, Klauber MR, Langer RD (1999) Quantitative and qualitative progression of peripheral arterial disease by non-invasive testing. Vasc Med 4:15-21

17. Reinhard H, Wiinberg N, Hansen PR et al (2011) NT-proBNP levels, atherosclerosis and vascular function in asymptomatic type 2 diabetic patients with microalbuminuria: peripheral reactive hyperaemia index but not NT-proBNP is an independent predictor of coronary atherosclerosis. Cardiovasc Diabetol 10:71

18. Tarnow L, Gall MA, Hansen BV, Hovind P, Parving HH (2006) Plasma N-terminal pro-B-type natriuretic peptide and mortality in type 2 diabetes. Diabetologia 49:2256-2262

19. Carter SA, Lezack JD (1971) Digital systolic pressures in the lower limb in arterial disease. Circulation 43:905-914

20. American Diabetes Association (2003) Peripheral arterial disease in people with diabetes. Diabetes Care 26:3333-3341

21. Leng GC, Fowkes FG, Lee AJ, Dunbar J, Housley E, Ruckley CV (1996) Use of ankle brachial pressure index to predict cardiovascular events and death: a cohort study. BMJ 313:1440-1444

22. Pencina MJ, D'Agostino RB, Vasan RS (2010) Statistical methods for assessment of added usefulness of new biomarkers. Clin Chem Lab Med 48:1703-1711

23. Hyun S, Forbang NI, Allison MA, Denenberg JO, Criqui MH, Ix JH (2014) Ankle-brachial index, toe-brachial index, and cardiovascular mortality in persons with and without diabetes mellitus. J Vasc Surg 60:390-395

24. Abbott JD, Lombardero MS, Barsness GW et al (2012) Anklebrachial index and cardiovascular outcomes in the bypass angioplasty revascularization investigation 2 diabetes trial. Am Heart J 164:585-590.e4

25. Ix JH, Miller RG, Criqui MH, Orchard TJ (2012) Test characteristics of the ankle-brachial index and ankle-brachial difference for medial arterial calcification on X-ray in type 1 diabetes. J Vasc Surg 56:721-727 